\title{
Galectin-9 regulates HTR8/SVneo function via JNK signaling
}

\author{
Mengdie Li ${ }^{1,2,3}$, Xiandong Peng ${ }^{2}$, Jinfeng Qian², Fengrun Sun², Chunqin Chen², Songcun Wang², \\ Jianping Zhang ${ }^{3}$ and Meirong Du(iD) ${ }^{1,2}$ \\ ${ }^{1}$ Department of Obstetrics and Gynecology, Guangzhou First People's Hospital, School of Medicine, South China \\ University of Technology, Guangzhou, China, ${ }^{2}$ Laboratory for Reproductive Immunology, NHC Key Lab of \\ Reproduction Regulation (Shanghai Institute of Planned Parenthood Research), Shanghai Key Laboratory of Female \\ Reproductive Endocrine Related Diseases, Hospital of Obstetrics and Gynecology, Fudan University Shanghai \\ Medical College, Shanghai, China and ${ }^{3}$ Department of Gynecology, Jing'an District Central Hospital, Fudan \\ University, Shanghai, China
}

Correspondence should be addressed to S Wang or J Zhang or M Du; Email: songcunwang@163.com or g5615335@126.com ordmrlq1973@sina.cn

\begin{abstract}
To obtain a successful pregnancy, trophoblasts must provide a physical barrier, suppress maternal reactivity, produce immunosuppressive hormones locally, and enhance the production of blocking factors that are able to bind to several antigenic sites. Inadequate placental perfusion has been closely associated with several pregnancy-associated diseases. Galectin-9 (Gal-9) has a wide variety of regulatory functions in innate and adaptive immunity during infection, tumor growth, and organ transplantation. We utilized immortalized human first-trimester extravillous trophoblast cells (HTR8/SVneo) for our functional study and examined the effects of Gal-9 on apoptosis, cytokine production and angiogenesis of HTR8/SVneo cells. Gal-9 inhibited the apoptosis and IFN- $\gamma$ and IL-17A production, promoted IL-4 production, and coordinated the crosstalk between HTR8/SVneo cells and human umbilical vein endothelial cells via its interaction with Tim-3. Blockade of JNK signaling inhibited Gal-9 activities in HTR8/SVneo cells. In addition, we detected a correlation between low levels of Gal-9 and spontaneous abortion. So Gal-9 could inhibit the apoptosis and proinflammatory cytokine expression, and promote the angiogenesis and IL-4 production in HTR8/SVneo cells via Tim-3 in a JNK dependent manner to help the maintenance of normal pregnancy. These findings possibly identify Gal-9 as a key regulator of trophoblast cells and suggest its potential as a biomarker and target for the treatment of recurrent pregnancy loss.

Reproduction (2021) $1611-10$
\end{abstract}

\section{Introduction}

The placenta is an important component of the maternal-fetal interface that invades the maternal uterus to provide nutrition and gas exchange for the developing fetus. Humans exhibit hemochorial placentation, the most invasive placental type in which trophoblast cells penetrate the maternal blood vessels and come into direct contact with maternal blood (Moffett \& Loke 2006). As a consequence of this process, tolerance mechanisms must be employed at the maternal-fetal interface to accept the semi-allogeneic fetus, while protecting both mother and fetus against infection. Disruption of this immune balance, however, causes the placenta and fetus to be attacked as a foreign organ transplant, resulting in pregnancy failure (Arck \& Hecher 2013). Extravillous trophoblasts (EVT) are an important cell type involved in the placentation process. In the human placenta, EVT invades the underlying decidua and migrates into the uterine spiral arteriolar walls, remodeling the uterine vasculature. Inadequate EVT invasion and placental perfusion have been closely associated with several pregnancy-associated diseases, including recurrent pregnancy loss (RPL), preeclampsia $(\mathrm{PE})$, and gestational trophoblastic diseases (AI-Khan et al. 2013).

Galectin-9 (Gal-9), a 36-kDa $\beta$-D-galactoside mammalian lectin, was identified from murine embryonic kidney and human Hodgkin's lymphoma tissues in 1997, and has a wide variety of regulatory functions in immunity (Fujita et al. 2017). Upon interaction with Tim-3 (receptor of Gal-9), Gal-9 terminates T helper 1 and $\mathrm{T}$ cytotoxic 1 cell responses, which plays a critical role in regulating immune activities during infection, tumor growth, and organ transplantation (Freeman et al. 2010). In metastatic melanoma, high soluble Gal-9 is correlated with T helper 2 (Th2) polarization and monocyte differentiation toward an M2 tumorpromoting phenotype (Enninga et al. 2016). In contrast to its immunosuppressive activities in promoting tumor 
escape, Gal-9 is also associated with cancer cell aggregation, apoptosis, and invasion inhibition (GoldenMason \& Rosen 2017).

In pregnancy, Gal-9 is secreted by trophoblast cells and can cause peripheral natural killer cells to take on a decidual NK phenotype via its receptor Tim-3 expressed on NK cells (Li et al. 2016, Sun et al. 2016). In addition, Lgals9 (Gal-9) mRNA levels in the placenta are higher than in the decidual, suggesting that Gal-9 could be important for placental development (Heusschen et al. 2013). However, the function of Gal-9 in placental development is poorly understood. In the present study, we investigated whether Gal-9 plays a functional role in the biological behaviors of trophoblast cells related to placenta development. We used immortalized human first-trimester extravillous trophoblast cells (HTR8/ SVneo) to perform functional studies. Given the pseudomalignant nature of the placenta, we examined the effects of Gal-9 in HTR8/SVneo function in the process of apoptosis, spiral artery remodeling and cytokine production. Here we showed that Gal-9 inhibited HTR8/SVneo apoptosis, while promoted tube formation of human umbilical vein endothelial cells (HUVECs) by HTR8/SVneo cells and regulated cytokine production by HTR8/SVneo cells. Furthermore, the expression of Gal-9 was decreased in RPL villus, implying that Gal-9 might play important roles in placentation and that aberrant expression of Gal-9 might be involved in the pathogenesis of RPL.

\section{Materials and methods}

\section{Human samples}

The use and collection of human samples were approved by the Human Research Ethics Committee of the Obstetrics and Gynecology Hospital of Fudan University. All participants provided written informed consent. All the methods were carried out in accordance with the approved guidelines. First-trimester (gestational age 6-12 weeks) human villus was obtained from clinical miscarriages (diagnosed as recurrent spontaneous abortion and excluding genetic, anatomic, or endocrine abnormalities or infection, poor health habits, etc., $n=31$ ) and normal pregnancies (terminated for non-medical reasons, $n=41$ ). The miscarried villous were obtained very soon after they were identified.

\section{Cell treatment}

HTR8/SVneo cells were grown in DMEM/Ham F12 medium supplemented with $10 \%$ fetal bovine serum, $100 \mathrm{U} / \mathrm{mL}$ penicillin, $100 \mu \mathrm{g} / \mathrm{mL}$ streptomycin, and $1 \mu \mathrm{g} / \mathrm{mL}$ amphotericin $\mathrm{B}$ at $37^{\circ} \mathrm{C}$ in $5 \% \mathrm{CO}_{2}$. HTR8/SVneo cells were cultured overnight in complete medium and further incubated in serumfree medium for $12 \mathrm{~h}$, followed by treatment with a range of concentrations of Gal-9 (clone 2045-GA, R\&D, 0, 10, 50, 100, 500,1000 , and $2500 \mathrm{ng} / \mathrm{mL}$, due to the limitations of human invasive experiments, we could not get the exact concentration of Gal-9 at the maternal-fetal interface at present and the median effective dose (ED50) of Gal-9 is $1-5 \mu \mathrm{g} / \mathrm{mL}$ ) for 48 h. To some wells, $10 \mu \mathrm{g} / \mathrm{mL}$ anti-Tim-3 mAb (clone F38-2E2; Biolegend, CA, USA), $10 \mu \mathrm{g} / \mathrm{mL}$ anti-Gal-9 mAb (clone 9M31, Biolegend), JNK signaling pathway inhibitor SP600125 $(15 \mu \mathrm{M})$, p38 signaling pathway inhibitor SB202190 (15 $\mu \mathrm{M})$, AKT inhibitor GDC0068 (15 $\mu \mathrm{M})$, or MEK1/2 inhibitor U0126 $(15 \mu \mathrm{M})$ was added.

HUVECs were grown in DMEM/Ham F12 medium supplemented with $10 \%$ fetal bovine serum, $100 \mathrm{U} / \mathrm{mL}$ penicillin, $100 \mu \mathrm{g} / \mathrm{mL}$ streptomycin, and $1 \mu \mathrm{g} / \mathrm{mL}$ amphotericin $\mathrm{B}$ at $37^{\circ} \mathrm{C}$ in $5 \% \mathrm{CO}_{2}$.

Trophoblasts cells were obtained from the villous tissue of normal pregnancies. The villous tissue was cut and digested in DMEM with high glucose and supplemented with trypsin $(0.25 \%)$ and DNase I (150 U/mL; Applichem, Germany) and underwent discontinuous Percoll gradient centrifugation as previously described (Wang et al. 2018). This method generates a $95 \%$ purity of trophoblast cells.

Trophoblasts cells were grown in DMEM with high glucose medium supplemented with $20 \%$ fetal bovine serum, 100 $\mathrm{U} / \mathrm{mL}$ penicillin, $100 \mu \mathrm{g} / \mathrm{mL}$ streptomycin, and $1 \mu \mathrm{g} / \mathrm{mL}$ amphotericin $\mathrm{B}$ at $37^{\circ} \mathrm{C}$ in $5 \% \mathrm{CO}_{2}$. Trophoblasts cells were cultured overnight in complete medium and further incubated in serum-free medium for $12 \mathrm{~h}$, followed by treatment with a range of concentrations of $1000 \mathrm{ng} / \mathrm{mL}$ Gal-9, To some wells $10 \mu \mathrm{g} / \mathrm{mL}$ anti-Tim-3 mAb, JNK signaling pathway inhibitor SP600125 (15 $\mu \mathrm{M})$ was added.

\section{Flow cytometry}

Alexa eFluor 488-conjugated anti-human IFN- $\gamma$, PE-conjugated anti-human activated caspase-3, PE/CY7-conjugated antihuman TNF- $\alpha$ or IL-10, APC-conjugated anti-human TGF- $\beta 1$, and brilliant violet 421-conjugated anti-human IL-4, IL-17A (Biolegend, CA, USA) were used. Cells were fixed and permeabilized using the Fix/Perm Kit (Biolegend, CA, USA). A minimum of 10,000 events were acquired using a CyAn ADP flow cytometer (Beckman-Coulter) and analyzed using Flowjo software (Tree Star, OR, USA).

\section{Annexin $V$ and 7AAD staining for cell apoptosis}

Freshly isolated HTR8/SVneo cells were seeded at $1 \times 10^{5}$ cells/well in 12-well plates and treated for $48 \mathrm{~h}$ with Gal-9 or antibodies as described earlier. The cells were harvested and resuspended in $100 \mu \mathrm{L}$ annexin-binding buffer containing $5 \mu \mathrm{L}$ PE-annexin $V$ and $1 \mu \mathrm{L}$ 7AAD working solution (BD Biosciences, San Jose, CA, USA) and then incubated in the dark for $15 \mathrm{~min}$ at room temperature. An additional 400 $\mu \mathrm{L}$ binding buffer was added, and HTR8/SVneo cells were analyzed immediately by flow cytometry (Beckman-Coulter).

\section{Tube formation assay}

In vitro cell tube formation was assayed by determining the ability of the cells to form tubes on a 3D Matrigel scaffold. Matrigel (356234, BD Biosciences) at a concentration of 9.9 $\mathrm{mg} / \mathrm{mL}$ was plated into 96 -well plates at $50 \mu \mathrm{L}$ per well and 
polymerized for $30 \mathrm{~min}$ at $37^{\circ} \mathrm{C}$. HUVECs stained with cell tracker red fluorescent probe (C2925, Invitrogen) were plated at $2.0 \times 10^{4}$ cells per well and HTR8/SVneo cells (treated for $48 \mathrm{~h}$ with Gal-9 or other antibodies as described earlier), which were stained with cell tracker green fluorescent probe (C2927, Invitrogen), were seeded at $2.0 \times 10^{4}$ cells per well in $100 \mu \mathrm{L}$ F12 medium. Tube formation was visualized under a bright-field microscope $4 \mathrm{~h}$ after implantation.

\section{Quantification of Gal-9 mRNA by real-time PCR}

The TRIzol reagent (Invitrogen Life Technologies) was used to extract RNA from the villus, and equal amounts of RNAs were reverse transcribed. The purity of the extracted RNA was tested photometrically, and the optical density (OD) at 260/280 nm was 1.8-2.0. The cDNAs were then amplified according to reverse kit (TaKaRa) instructions. A $20 \mu \mathrm{L}$ reaction contained 1 $\mu \mathrm{g}$ template RNA, $4 \mu \mathrm{L}$ of $5 \times$ PrimeScript RT Master Mix and the rest of $20 \mu \mathrm{L}$ was RNase Free $\mathrm{dH}_{2} \mathrm{O}$. Reactions were incubated at $37^{\circ} \mathrm{C}$ for $15 \mathrm{~min}, 85^{\circ} \mathrm{C}$ for $5 \mathrm{~s}$, and then were instantly cooled on ice. The primers were designed with computer assistance according to GenBank sequences. The primers for Gal-9 were as follows: 5'-CCACCTGACCAGAGTGTTCTCT-3' (forward) and 5'-ATGAAAATGCTTGTTGGCACATT-3' (reverse). Each expression level was normalized to the housekeeping gene $\beta$-actin, using the primers 5'-GCCGACAGGATGCAGAAGGAGATCA-3' (forward) and 5'-AAGCATTTGCGGTGGACGATGGA-3' (reverse). Real-time PCR was performed in a final volume of $20 \mu \mathrm{L}$ containing 10 $\mu \mathrm{L}$ of SYBR Green Master Mix (Yeasen, Shanghai, China), 0.8 $\mu \mathrm{L}$ of $2.5 \mathrm{mM}$ primer, $2 \mu \mathrm{L}$ DNA template, and $7.2 \mu \mathrm{L}$ distilled $\mathrm{H}_{2} \mathrm{O}$. The PCR was implemented according to the following parameters: $95^{\circ} \mathrm{C}$ for $5 \mathrm{~min}, 40$ cycles at $95^{\circ} \mathrm{C}$ for $10 \mathrm{~s}, 60^{\circ} \mathrm{C}$ for $30 \mathrm{~s}, 72^{\circ} \mathrm{C}$ for $20 \mathrm{~s}$.

\section{Western blot analysis}

HTR8/SVneo cells were stimulated by $1000 \mathrm{ng} / \mathrm{mL}$ Gal-9 for $0,15,30,60,90$, or $120 \mathrm{~min}$. The Cells were harvested and homogenized in an ice-cold radioimmunoprecipitation assay (RIPA) lysis buffer (Beyotime, Shanghai, China). Total cell lysates were collected, and equal quantities of protein were separated by SDS-PAGE and blotted onto polyvinylidene difluoride (PVDF) membranes. The PVDF membranes were blocked with Tris-buffered saline (TBS) containing 5\% skimmed milk powder for $1 \mathrm{~h}$ at room temperature and incubated with anti-phospho-JNK (1:1000, Cell Signaling Technology), antiJNK (1:1000, Cell Signaling Technology) or anti-GAPDH mAb (1:250, Santa Cruz Biotechnology) overnight at $4{ }^{\circ} \mathrm{C}$. Then, the membranes were washed with $1 \times$ TBS/Tween 20 buffer three times and incubated with horseradish-peroxidase-conjugated polyclonal secondary antibodies for $1 \mathrm{~h}$ at room temperature. The membranes were developed with the enhanced plus chemiluminescence assay.

Villi were homogenized in RIPA buffer. Equal amounts of proteins were subjected to $10 \%$ SDS-PAGE and electrophoretically transferred to PVDF membranes. The PVDF membranes were blocked with Tris-buffered saline (TBS) containing 5\% skimmed milk powder for $1 \mathrm{~h}$ at room temperature and incubated with anti-Gal-9 (1:1000, Abcam), or anti- $\beta$-Actin mAb (1:1000, Beyotime, Shanghai, China) overnight at $4^{\circ} \mathrm{C}$. Then, the membranes were washed with $1 \times \mathrm{TBS} /$ Tween 20 buffer three times and incubated with horseradish-peroxidase-conjugated polyclonal secondary antibodies for $1 \mathrm{~h}$ at room temperature. The membranes were developed with the enhanced plus chemiluminescence assay.

\section{Immunohistochemical analysis}

Sections $(5 \mathrm{~mm})$ of paraffin-embedded first-trimester human villus were rehydrated in Tris-buffered saline (TBS) and incubated with hydrogen peroxide and 1\% BSA to block endogenous peroxidase activity. Sections were incubated with rabbit anti-human Gal-9 antibody $(0.5 \mathrm{mg} / \mathrm{mL}$, ab69630; Abcam) overnight in a humidified chamber. After washing, sections were overlaid with poly-HRP anti-rabbit IgG (Golden Bridge International, Beijing, China). The reaction was developed with 3,3-diaminobenzidine (DAB), and sections were counterstained with hematoxylin.

\section{Statistical analysis}

All variables were normally distributed in this study. Thus, variables were presented as means and S.D. One-way ANOVA was used to evaluate differences. A $P$ value of less than 0.05 was considered statistically significant. For variables with a $P$ value of less than 0.05 in ANOVA, the post hoc Dunnett $t$-test was performed to determine differences between each group. All analyses were carried out using the GraphPad Prism 5 software.

\section{Results}

\section{Gal-9 regulated biological behaviors of HTR8/ SVneo cells}

To identify the specific roles of Gal-9 in placentation, here we focused on how Gal-9 affected the apoptosis, tube forming ability and cytokine production of HTR8/ SVneo cells, as Gal-9 could promote HTR8/SVneo cell invasion and had no effect on the viability or proliferation of HTR8/SVneo cells (data not shown). As shown in Fig. $1 \mathrm{~A}$, with a concentration range of $10-2500 \mathrm{ng} / \mathrm{mL}$, Gal-9 inhibited the apoptosis of HTR8/SVneo cells in a concentration-dependent manner. And this apoptosis inhibition may be achieved by reducing the activation of Caspase-3 (Fig. 1B).

A polarization toward Th2 bias and the balance between regulatory (Treg) and Th17 in the immune milieu at the maternal-fetal interface have long been considered the main mechanism of maternal-fetal tolerance (Figueiredo \& Schumacher 2016, Sheu et al. 2018). We next directly analyzed the related cytokine production of HTR8/SVneo cells treated with Gal-9. We found that Gal-9 decreased HTR8/SVneo production of proinflammatory Th1 cytokine IFN- $\gamma$ and Th17 cytokine IL-17A, and increased anti-inflammatory Th2 cytokine 
A

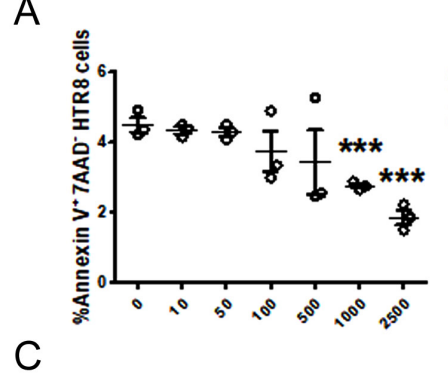

$\mathbf{0}$

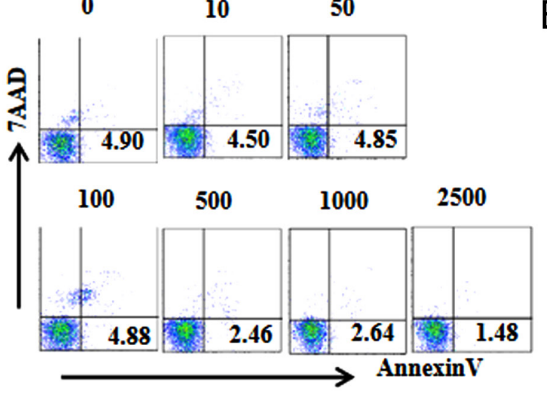

$B \stackrel{0}{\overline{0}}$



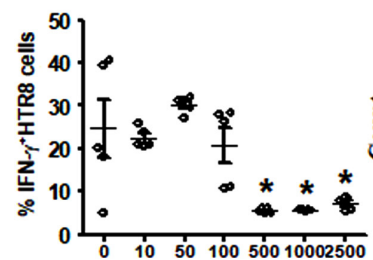
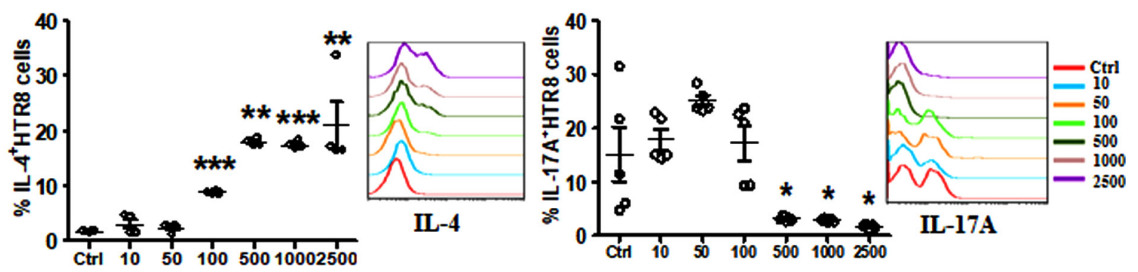

D

Ctrl

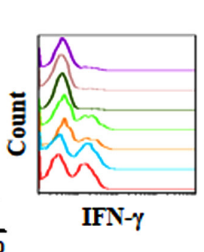

10

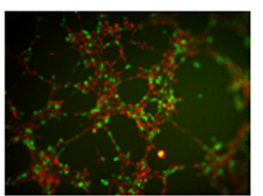

100

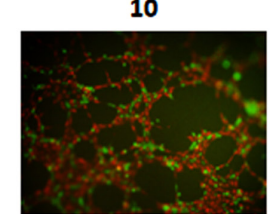

1000

500
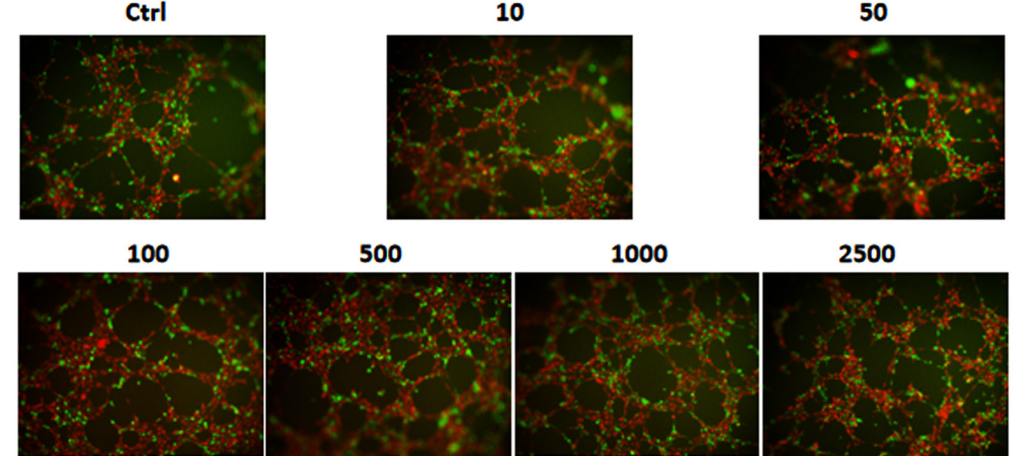

2500

HUVECS

HTR8/SVneo

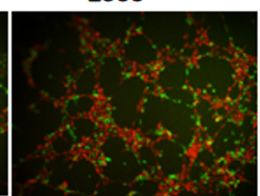

Figure 1 Gal-9 regulated biological behaviors of HTR8/SVneo cells. (A) HTR8/SVneo cells were seeded at $1 \times 10^{5}$ cells $/ \mathrm{mL}$ in 12 -well plates and treated with a range of concentrations of Gal-9 (0, 10, 50, 100, 500, 1000, and $2500 \mathrm{ng} / \mathrm{mL})$ for $48 \mathrm{~h}$. A PE Annexin V apoptosis detection kit was used to analyze HTR8/SVneo cell apoptosis. (B) Flow cytometric analysis assessing the activated caspase-3 of HTR8/SVneo cells treated with or without (Ctrl) Gal-9. (C) Flow cytometric analysis assessing the expression of IFN- $\gamma$, IL-4 and IL-17A by HTR8/SVneo cells treated with or without (Ctrl) Gal-9. Data represent mean \pm s.E.M. ${ }^{* * *} P \leq 0.001$. (D) Immunofluorescent assay for the 3D tubes formed by HUVECs (red) and HTR8/SVneo cells (green, treated with or without (Ctrl) Gal-9) at $4 \mathrm{~h}$. Images are representative of three individual experiments.

IL-4 (Fig. 1B), but had no effect on the production of TNF- $\alpha$, IL-10 and TGF- $\beta 1$ (Supplementary Fig. 1, see section on supplementary materials given at the end of this article).

During spiral artery remodeling, EVT interacts with endothelial cells directly. Here, the tube formation ability of HUVECs and HTR8/Svneo cells (following treatment with a concentration range of Gal-9) coculture system was observed by a tube formation assay. The results showed that Gal-9 promoted tube formation by HUVECs and HTR8/SVneo cells coculture system, indicating that Gal-9 could promote angiogenesis directly.

All these effects were observed when HTR8/SVneo cells were treated with Gal-9 at a concentration of 1000 $\mathrm{ng} / \mathrm{mL}$, and we noticed that the effect of $2500 \mathrm{ng} / \mathrm{mL}$ Gal-9 was weaker than that of $1000 \mathrm{ng} / \mathrm{mL}$ Gal-9 in tube formation assay; therefore, we used the concentration $1000 \mathrm{ng} / \mathrm{mL}$ in the follow-up experiments.

\section{Gal-9 regulated biological behaviors of HTR8/SVneo cells by interacting with Tim-3}

Previousstudies haveindicated thattheimmunoregulatory effect of Gal-9 depends on its interaction with its receptor CD44 or Tim-3 (Golden-Mason \& Rosen 2017). Therefore, we used anti-CD44 or anti-Tim-3 neutralizing antibodies to analyze the Gal-9 interaction with these receptors in HTR8/SVneo cells. We found that anti-CD44 mAb had no effect on Gal-9-mediated biological behaviors regulation (data not shown). Pretreatment with anti-Tim-3 mAb notably reversed the inhibition of HTR8/SVneo apoptosis by Gal-9 (Fig. 2A and B). Anti-Tim-3 mAb also reversed the effect of Gal-9 on cytokine production and tube formation (Fig. 2C and D). And the effects of Tim-3 blockade were comparable to Gal-9 blockade (Fig. 2). These observations indicated that the activity of Gal-9 was dependent on Tim-3, rather than CD44, in HTR8/SVneo cells. 
A



B

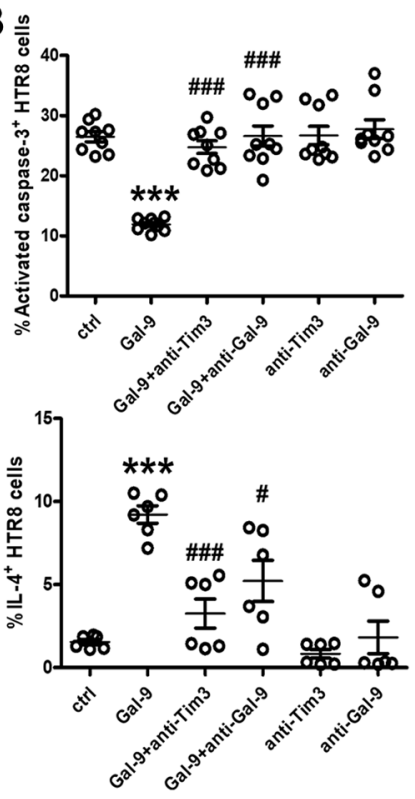

D

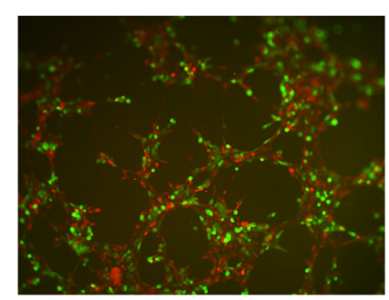

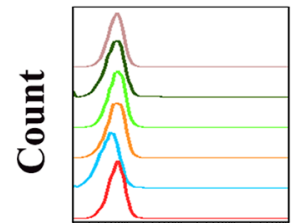

Activated caspase-3

C

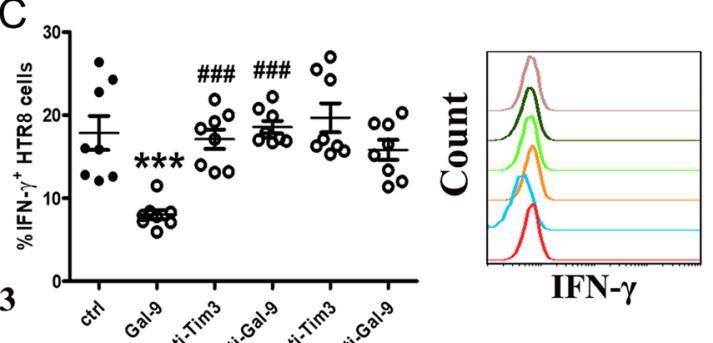

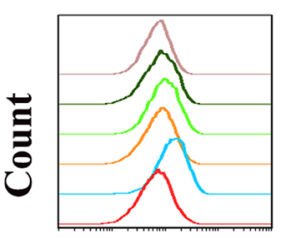

IL-4

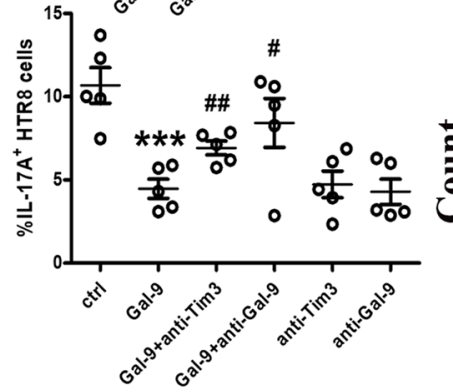

Gal-9 + anti-Tim3

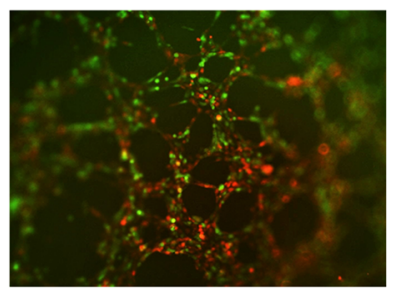

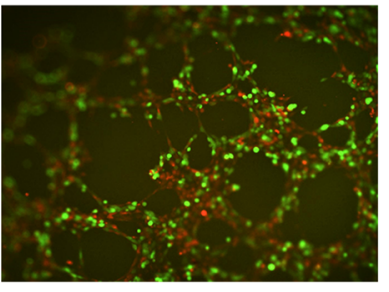

Figure 2 Gal-9 regulated biological behaviors of HTR8/SVneo cells in a Tim-3-dependent manner. (A, B and C) Flow cytometric analysis and quantitation of apoptosis (A), activated caspase-3 (B) and cytokine expression (C) of HTR8/SVneo cells after stimulation with or without Gal-9 $(1000 \mathrm{ng} / \mathrm{mL})$ in the presence or absence of anti-Gal-9, or anti-Tim-3 mAbs for $48 \mathrm{~h}$. Flow cytometry plot is from one representative experiment. Data represent mean \pm S.E.M. ${ }^{* *} P \leq 0.01,{ }^{* * *} P \leq 0.001$, compared with the group Ctrl. ${ }^{\sharp} P<0.05,{ }^{\sharp \#} P<0.01$, ${ }^{\sharp \sharp} P \leq 0.001$ compared with the Gal-9 group. (D) Tube formation assay of HUVECs (red) and HTR8/SVneo cells (green, after stimulation with or without Gal-9 in the presence or absence of anti-Gal-9, or anti-Tim-3 mAbs) at $4 \mathrm{~h}$. Images are representative of three individual experiments.

\section{Gal-9 regulated biological behaviors of HTR8/SVneo cells depending on JNK signaling}

To further investigate the signaling pathways involved in Gal-9-mediated biological behaviors regulation of HTR8/SVneo cells, the effect of specific signal transduction inhibitors on HTR8/SVneo cell apoptosis was examined. The results showed that most signaling pathways inhibitors, including GDC0068 (inhibitor of AKT), SB202190 (inhibitor of p38 mitogen-activated protein kinase) and U0126 (inhibitor of ERK1/2) had no effect on Gal-9-mediated apoptosis inhibition. However, SP600125 (inhibitor of JNK) markedly reversed this effect (Fig. 3A and B). Additionally, treatment with SP600125 alone did not affect HTR8/SVneo cell 
A

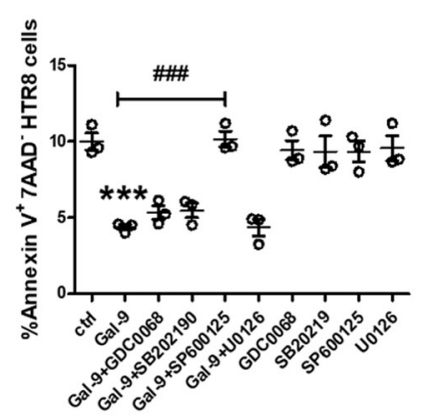

B $\frac{\underline{\underline{x}}}{\overline{8}}$



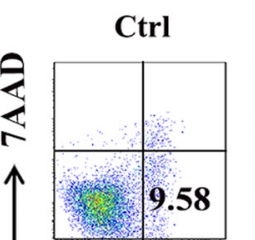

Gal-9

Gal-9
$+\mathbf{U 0 1 2 6}$

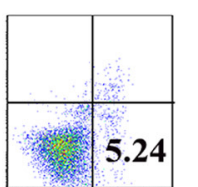

5.24
Gal-9

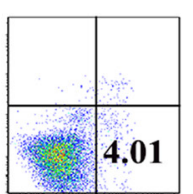

GDC0068
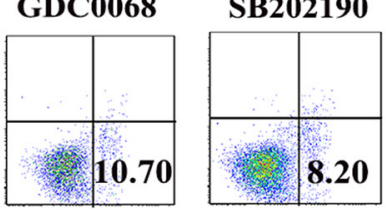

8.20

+GDC0068

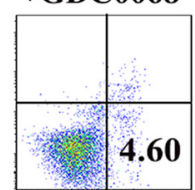

4.60

Gal-9
+ SB20219

+SB202190

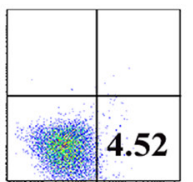

4.52

SP600125

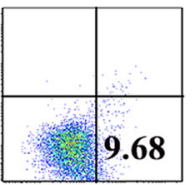

9.68

C

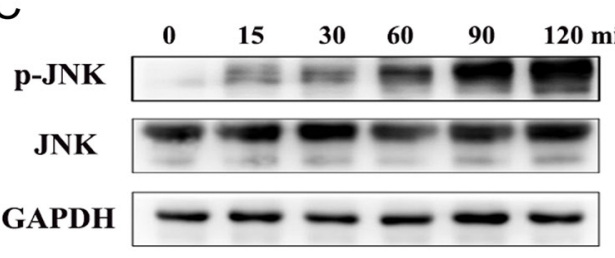


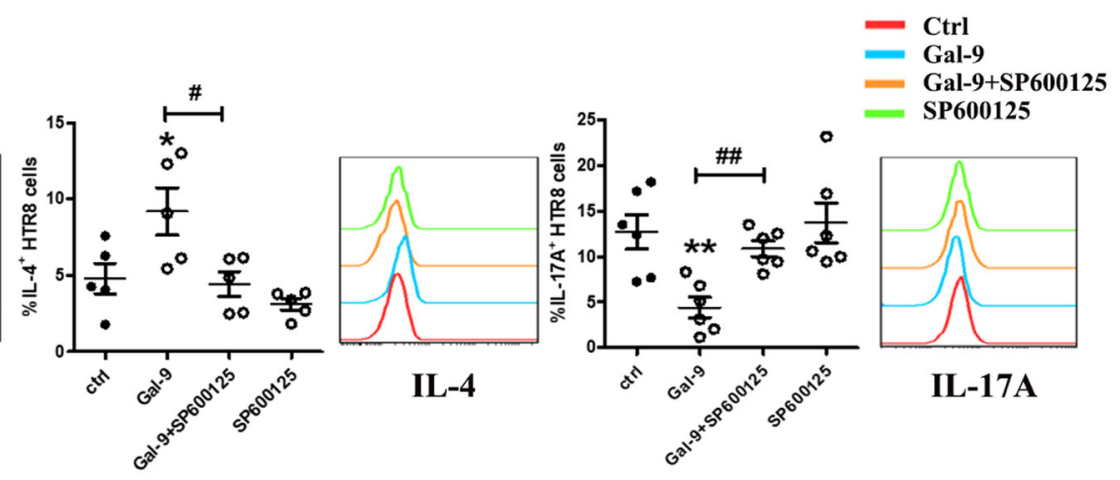

E

Ctrl

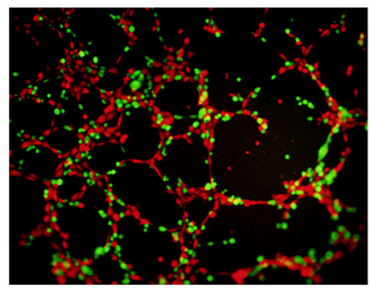

Gal-9



Gal-9+SP600125

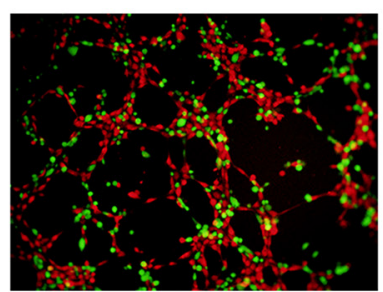

8.39

\section{AnnexinV}

Gal-9

+SP600125

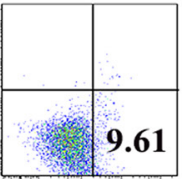

U0126

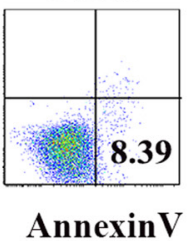

$\circ$

IL-17A

Figure 3 Gal-9 regulated biological behaviors of HTR8/SVneo cells depending on JNK signaling. (A and B) Flow cytometric analysis of HTR8/SVneo cells apoptosis after stimulation with or without Gal-9 $(1000 \mathrm{ng} / \mathrm{mL})$ in the presence or absence of the specific signaling pathway inhibitors for $48 \mathrm{~h}$. The flow cytometry plot is from one representative experiment. Data represent mean \pm S.E.M. ${ }^{* * *} P \leq 0.001$, compared with the group Ctrl. ${ }^{\sharp \# P}<0.001$, compared with the Gal-9 group. (C) HTR8/SVneo cells were treated with $1000 \mathrm{ng} / \mathrm{mL}$ Gal-9 at a different time. A Western blot was used to analyze the total levels and phosphorylation levels of JNK. Gal-9 induced JNK phosphorylation in HTR8/SVneo cells in a time-dependent manner. The image is representative of four individual experiments. (D) Flow cytometric analysis of cytokine expression by HTR8/SVneo cells after stimulation with or without Gal-9 $(1000 \mathrm{ng} / \mathrm{mL})$ in the presence or absence of the SP600125 for $48 \mathrm{~h}$. The flow cytometry plot is from one representative experiment. Data represent mean \pm S.E.M. ${ }^{*} P \leq 0.05,{ }^{* *} P \leq 0.01,{ }^{* * *} P \leq 0.001$, compared with the group Ctrl. ${ }^{\sharp} P<0.05,{ }^{\sharp} P<0.01,{ }^{\sharp \#} P \leq 0.001$ compared with the Gal-9 group. (E) Tube formation assay of HUVECs (red) and HTR8/SVneo cells (green, after stimulation with or without Gal-9 in the presence or absence of SP600125) at $4 \mathrm{~h}$. Images are representative of three individual experiments. 
A

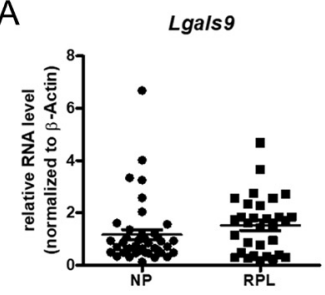

B
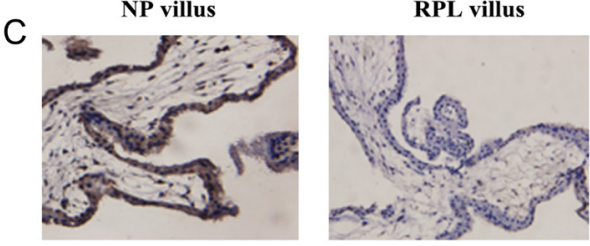

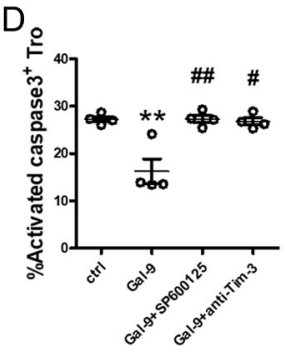

E
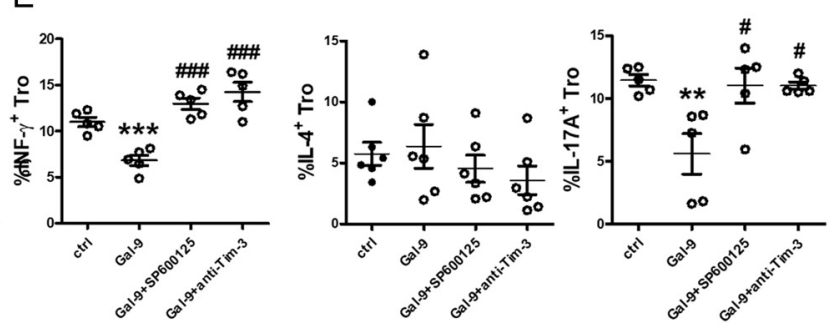

Figure 4 The protein level of Gal-9 was decreased in the villus of RPL patients. (A) Real-time PCR analysis of Gal-9 mRNA in human first-trimester villus from normal pregnancy $(n=15)$ and RPL patients $(n=12)$. (B) Western blot analysis of Gal-9 in human first-trimester villus from normal pregnancy $(n=15)$ and RPL patients $(n=12)$. (C) Immunohistochemical localization of Gal-9 in villus from pregnancy $(n=15)$ and miscarriage $(n=12)$. (D and E) Flow cytometric analysis assessing the activated caspase-3 (D) and cytokine expression (E) of Trophoblast cells after stimulation with or without Gal-9 $(1000 \mathrm{ng} / \mathrm{mL})$ in the presence or absence of an anti-Tim-3 mAb or SP600125 for 48 h. Data represent the mean \pm S.E.M. of one representative experiment among three to five separate experiments. ${ }^{* *} P \leq 0.01$,

${ }^{* * *} P \leq 0.001$, compared with the group Ctrl. ${ }^{\sharp} P<0.05,{ }^{\sharp} P<0.01,{ }^{\sharp \#} P \leq 0.001$ compared with the Gal-9 group. apoptosis, suggesting the inhibition of apoptosis caused by SP600125 resulted in a reverse of the Gal-9-mediated activity.

Next, we analyzed whether Gal-9 activated JNK signaling in HTR8/SVneo cells. As shown in Fig. 3C, the administration of Gal-9 significantly increased JNK phosphorylation, sustaining for at least $120 \mathrm{~min}$ (Fig. 3C). Then we further examined whether SP600125 could reversed the effects of Gal-9 on cytokine production and tube formation. The results showed that SP600125 blocked Gal-9-induced IL-4 production and angiogenesis, while reversed the decrease of IFN- $\gamma$ and IL-17A (Fig. 3D and E). These findings suggested that Gal-9 regulated HTR8/SVneo cell biological behaviors might depend on the JNK signaling pathway.

\section{The protein level of Gal-9 was decreased in the villus of RPL patients}

Since Gal-9 had been proved to be good for maintaining normal function of EVT, including invasion (data not shown), apoptosis, tube formation and cytokine production. We further explored whether Gal-9 was involved in the pathogenesis of pregnant complications attributed to placental insufficiency, such as RPL. Surprisingly, we found that the mRNA level of Gal-9 did not decrease in the villus of RPL patients, which was consistent with a previous study between the abortion-prone and normal pregnant mouse model (He et al. 2018) (Fig. 4A). However, Gal-9 protein was significantly decreased in RPL villus by western blot (Fig. 4B). Importantly, IHC analysis of villus also showed that Gal-9 protein was reduced in the RPL villus (Fig. 4C).

To explore the effects of Gal-9 on primary cells during pregnancy, we treated trophoblast cells with Gal-9,
anti-Tim-3 mAb, and SP600125. As shown in Fig. 4D and E, Gal-9 also down-regulated the expression of apoptosis marker activated caspase-3, and proinflammatory cytokines (IFN- $\gamma$ and IL-17A) in a Tim-3 and JNK dependent manner, but had no effect on the expression of IL-4 in primary trophoblast cells. These data imply that Gal-9 might play an important role in placentation and maternal-fetal tolerance.

Figure 5 presented the proposed mechanism of how Gal-9 regulation the biological function of HTR8/SVneo cells.

\section{Discussion}

Successful pregnancy relies on sufficient placental formation and the maternal immune system tolerating the semi-allogeneic fetus. Adequate EVT function is vital in placental development, and placental insufficiency is closely related to pregnancy complications, such RPL and PE. As miscarriage is the most common complication of pregnancy, and PE is a leading cause of maternal and fetal morbidity and mortality (Shennan et al. 2017), improving the biological function of trophoblasts is highly important and has raised the concerns of many scientists.

To obtain a successful pregnancy, trophoblasts must (i) provide a physical barrier, (ii) suppress maternal reactivity, (iii) produce immunosuppressive, hormones locally, and (iv) enhance the production of blocking factors that are able to bind to several antigenic sites (He et al. 2018). In the present study, we provided the first insight of how Gal-9 regulated the biological function of HTR8/SVneo cells and the crosstalk between trophoblasts and endothelial cells. Gal-9 inhibited the apoptosis and proinflammatory cytokine production 


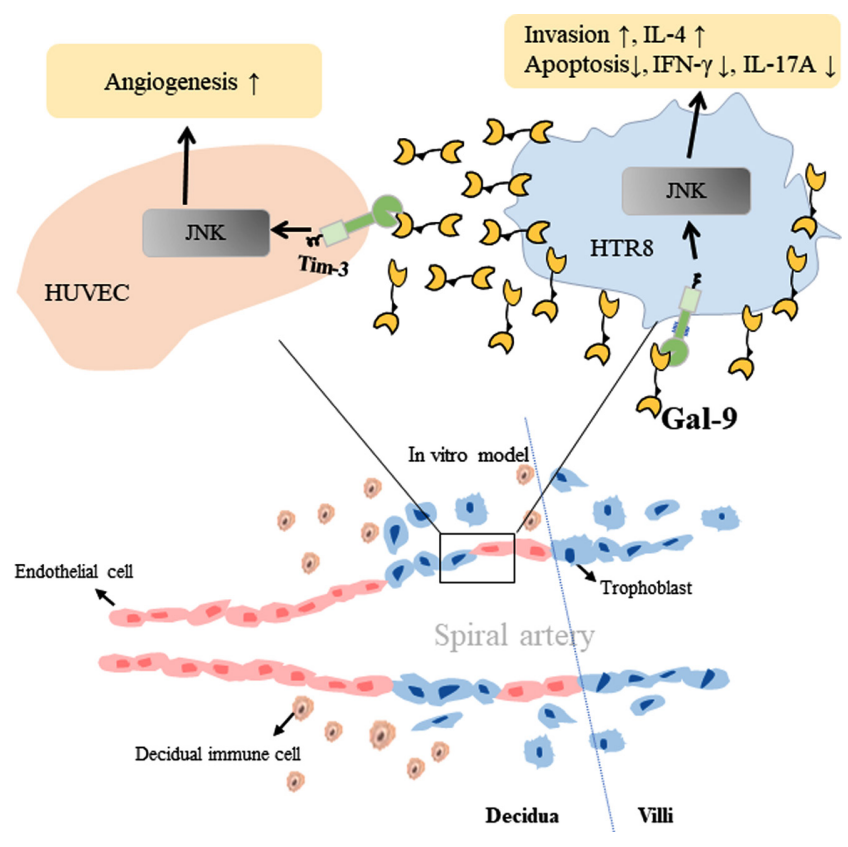

Figure 5 Schematic diagram of Gal-9-mediated regulation of human trophoblast cell biological behaviors. Trophoblast cells secrete Gal-9, which in turn interacts with its receptor Tim-3 in trophoblast cells. Interaction of Gal-9/Tim-3 activates the JNK signaling pathway, which inhibits the apoptosis and IFN- $\gamma$ and IL-17A production, and promotes the tube formation, invasion and IL-4 production in trophoblast cells via Tim-3 in a JNK dependent manner.

of HTR8/SVneo cells, while coordinating the crosstalk between HTR8/SVneo cells and endothelial cells to promote the tube formation. In addition, Gal-9 was found to be down-regulated in the villus of RPL patients. Therefore, our results suggest that Gal-9 expression in trophoblasts might be critical for a normal pregnancy.

Gal-9 is currently known as a versatile immunomodulator that affects a host of cell types, including leukocytes, stromal cells and endothelial cells. In previous studies, our team confirmed that Tim3, a receptor of Gal-9, formed a negative feedback loop to inhibit TLR-triggered inflammatory responses in decidual stromal cells (Wang et al. 2015). And Gal-9 was a novel endometrial marker for the mid- and latesecretory and decidual phases (Popovici et al. 2005). Compared to non-pregnant individuals, regulatory T cells showed a higher level of Gal-9 expression as pregnancy proceeding, Gal-9-expressing regulatory $\mathrm{T}$ cells played an important role in the maintenance of a healthy pregnancy (Meggyes et al. 2014). Gal-9 could alleviate LPS-induced preeclampsia-like impairment in rats via switching decidual macrophage polarization to M2 subtype (Li et al. 2018). However, the function of Gal-9 in placentation is poorly understood.

In previous studies, we confirmed that Gal-9 promotes HTR8/SVneo cell invasion. In the present study, we further found that Gal-9 could also inhibit
HTR8/SVneo cell apoptosis. One novel finding of the present study is the critical role of Gal-9 involved in the crosstalk between HTR8/SVneo cells and HUVECs. We gave the direct evidence that Gal-9 coordinated the crosstalk between HTR8/SVneo cells and endothelial cells to promote the tube formation. These findings further illuminated that Gal-9 might play vital roles in a placental formation.

It is widely believed that a polarization toward Th2 bias and the balance between Treg and Th17 at the maternal-fetal interface were important for maternalfetal tolerance (Arck \& Hecher 2013, Figueiredo \& Schumacher 2016). These studies are mostly presented in the maternal immune system, reveling that the maternal immune system tolerating the semi-allogeneic fetus. Previous studies, including ours, have revealed that trophoblasts had the unique ability to instruct decidual immune cells to develop a regulatory phenotype for fetal tolerance (Svensson-Arvelund et al. 2015, Wang et al. 2019). We found that Gal-9 could increase the production of IL-4, while decreasing the production of IFN- $\gamma$ and IL-17A in HTR8/SVneo cells. In another study, Gal-9 decreased production of Th1-type cytokines, and triggered a Th2 bias in cocultures of trophoblast cells from abortion-prone mice (He et al. 2018). We speculate that Gal-9 might therefore also be used as an important marker to indicate the tolerant state of the maternal milieu. Gal-9 thereby serves as an immunetolerant subset. Since Gal-9 has a critical regulatory role in immune cell function during pregnancy (Li et al. 2017, He et al. 2018) and decidual immune cells are vital for the immune regulation of uterine spiral arteries remodeling (Blois et al. 2011), it is possible that the Gal-9 produced by trophoblasts also regulates placenta development by affecting the function of decidual immune cells.

We further confirmed that the activity of Gal-9 in HTR8/SVneo cells was dependent on Tim-3, similar to most immune cells. The JNK protein, a kinase belonging to the MAPK superfamily, modulates diverse cellular events and is of great importance for angiogenesis, proliferation, and invasion (O'Brien et al. 2018). MAPK superfamily could also activate a variety of transcription factors that coordinate the induction of a number of genes encoding for inflammatory mediators as well as anti-inflammatory cytokines (Kim et al. 2010). Blockade of JNK signaling inhibited Gal-9-induced IL-4 production and tube formation, while reversed the apoptosis inhibition and IFN- $\gamma$ and IL-17A production in HTR8/ SVneo cells. A similar phenomenon has been observed showing that an inhibitor of JNK signaling can block the effects of Gal-9 on the differentiation of Th1 and Th17cells and the cytoprotection by human umbilical cord-derived mesenchymal stem cells (Fan et al. 2018).

Hutter et al. reported that there was significant down-regulation of Gal-9 in the fetal growth restriction 
(FGR) trophoblast of male fetuses. In contrast, in FGR pregnancies with female fetus, Gal-9 was upregulated in the EVT (Hutter et al. 2015). The Gal-9 protein levels were significantly decreased in the LPS-induced PE-like rats (Li et al. 2018). In our studies, we found there was a significantly enhanced Gal-9 expression on villi by normal groups compared with RPL patients. As such, abnormal Gal-9 expression could be an early warning sign of adverse pregnancy outcome.

In summary, we found that Gal-9 could inhibit the apoptosis and IFN- $\gamma$ and IL-17A production in HTR8/SVneo cells and trophoblast cells via Tim-3 in a JNK dependent manner, and promote the tube formation and IL-4 production in HTR8/SVneo cells (Fig. 5). Gal-9 had no effect on IL-4 expression on trophoblast cells, suggesting that though HTR8/SVneo cells have been proven effective for recapitulating key aspects of primary trophoblast cells, immortalized cell lines could not represent the in vivo environment exactly. According to our hypothesis, the dominant presence of Gal-9 on trophoblast cells at the maternal-fetal interface suggests a subsequent local immunosuppressive and angiogenic potential. Our data has expanded the regulatory function of the Gal-9/Tim-3 signaling pathway on maternal-fetal crosstalk and has provided a potential biomarker or/ and therapeutic target for RPL.

\section{Supplementary materials}

This is linked to the online version of the paper at https://doi. org/10.1530/REP-19-0543.

\section{Declaration of interest}

The authors declare that there is no conflict of interest that could be prejudice the impartiality of the research reported.

\section{Funding}

This work was supported by grant from the National Key R\&D Program of China (2017YFC1001403 to M R D and 2017YFC1001404 to D J L), the Nature Science Foundation from National Nature Science Foundation of China (NSFC) (31700799 to S C W, 31970859, 81630036, 91542116 and 31570920 to M R D), the Program of Shanghai Academic/ Technology Research Leader (17XD1400900 to M R D), the Innovation-oriented Science and Technology Grant from NPFPC Key Laboratory of Reproduction Regulation (CX2017-2 to M R D), the Shanghai Sailing Program (17YF1411600 to S C W), the Training Program for Young Talents of Shanghai Health System (2018YQ07 to S C W), the Shanghai Chenguang Program (18CG09 to S C W) and Development Fund of Shanghai Talents (2018110 to S C W). None of the authors have any conflict of interest to declare.

\section{Author contribution statement}

S C W and M R D conceived experiments. S C W and M D L carried out experiments and analyzed data. J F Q, M D L, F R S, C Q C coordinated the sample collection, data interpretation, literature search, and figure preparation. S C W drafted the manuscript. J P Z and $M R D$ revised the manuscript. All authors reviewed the manuscript.

\section{References}

Al-Khan A, Bulmer JN, Chantraine F, Chen CP, Chen Q, Collins S, Cotechini T, Fitzgerald JS, He M, Holland O et al. 2013 IFPA Meeting 2012 Workshop Report III: trophoblast deportation, gestational trophoblastic disease, placental insufficiency and fetal growth restriction, trophoblast over-invasion and accreta-related pathologies, placental thrombosis and fibrinolysis. Placenta 34 (Supplement) S11-S16. (https:// doi.org/10.1016/j.placenta.2012.11.018)

Arck PC \& Hecher K 2013 Fetomaternal immune cross-talk and its consequences for maternal and offspring's health. Nature Medicine 19 548-556. (https://doi.org/10.1038/nm.3160)

Blois SM, Klapp BF \& Barrientos G 2011 Decidualization and angiogenesis in early pregnancy: unravelling the functions of DC and NK cells. Journal of Reproductive Immunology 88 86-92. (https://doi.org/10.1016/j. jri.2010.11.002)

Enninga EA, Nevala WK, Holtan SG, Leontovich AA \& Markovic SN 2016 Galectin-9 modulates immunity by promoting Th2/M2 differentiation and impacts survival in patients with metastatic melanoma. Melanoma Research 26 429-441. (https://doi.org/10.1097/ CMR.0000000000000281)

Fan J, Tang X, Wang Q, Zhang Z, Wu S, Li W, Liu S, Yao G, Chen H \& Sun L 2018 Mesenchymal stem cells alleviate experimental autoimmune cholangitis through immunosuppression and cytoprotective function mediated by galectin-9. Stem Cell Research and Therapy 9 237. (https:// doi.org/10.1186/s13287-018-0979-x)

Figueiredo AS \& Schumacher A 2016 The T helper type 17/regulatory T cell paradigm in pregnancy. Immunology 148 13-21. (https://doi. org/10.1111/imm.12595)

Freeman GJ, Casasnovas JM, Umetsu DT \& DeKruyff RH 2010 TIM genes: a family of cell surface phosphatidylserine receptors that regulate innate and adaptive immunity. Immunological Reviews 235 172-189. (https:// doi.org/10.1111/j.0105-2896.2010.00903.x)

Fujita K, Iwama H, Oura K, Tadokoro T, Samukawa E, Sakamoto T, Nomura T, Tani J, Yoneyama H, Morishita A et al. 2017 Cancer therapy due to apoptosis: galectin-9. International Journal of Molecular Sciences 18 74. (https://doi.org/10.3390/ijms18010074)

Golden-Mason L \& Rosen HR 2017 Galectin-9: diverse roles in hepatic immune homeostasis and inflammation. Hepatology 66 271-279. (https://doi.org/10.1002/hep.29106)

He M, Jiang M, Zhou Y, Li F, Yang M, Fan Y, Xie Y, Beejadhursing R, Feng L \& Deng D 2018 Impaired Gal-9 dysregulates the PBMC-induced Th1/ Th2 imbalance in abortion-prone matings. Journal of Immunology Research 2018 9517842. (https://doi.org/10.1155/2018/9517842)

Heusschen R, Freitag N, Tirado-Gonzalez I, Barrientos G, Moschansky P, Munoz-Fernandez R, Leno-Duran E, Klapp BF, Thijssen VL \& Blois SM 2013 Profiling Lgals9 splice variant expression at the fetalmaternal interface: implications in normal and pathological human pregnancy. Biology of Reproduction 88 22. (https://doi.org/10.1095/ biolreprod.112.105460)

Hutter S, Knabl J, Andergassen U, Mayr D, Hofmann S, Kuhn C, Mahner S, Arck P \& Jeschke U 2015 Fetal gender specific expression of tandemrepeat galectins in placental tissue from normally progressed human pregnancies and intrauterine growth restriction (IUGR). Placenta 36 1352-1361. (https://doi.org/10.1016/j.placenta.2015.09.015)

Kim JY, Cho MK, Choi SH, Lee KH, Ahn SC, Kim DH \& Yu HS 2010 Inhibition of dextran sulfate sodium (DSS)-induced intestinal inflammation via enhanced IL-10 and TGF-beta production by galectin-9 homologues isolated from intestinal parasites. Molecular and Biochemical Parasitology 174 53-61. (https://doi.org/10.1016/j. molbiopara.2010.06.014) 
Li YH, Zhou WH, Tao Y, Wang SC, Jiang YL, Zhang D, Piao HL, Fu Q, Li DJ \& Du MR 2016 The galectin-9/Tim-3 pathway is involved in the regulation of NK cell function at the maternal-fetal interface in early pregnancy. Cellular and Molecular Immunology 13 73-81. (https://doi. org/10.1038/cmi.2014.126)

Li Y, Zhang J, Zhang D, Hong X, Tao Y, Wang S, Xu Y, Piao H, Yin W \& Yu M 2017 Tim-3 signaling in peripheral NK cells promotes maternalfetal immune tolerance and alleviates pregnancy loss. Science Signaling 10 eaah4323.

Li ZH, Wang LL, Liu H, Muyayalo KP, Huang XB, Mor G \& Liao AH 2018 Galectin-9 alleviates LPS-induced preeclampsia-like impairment in rats via switching decidual macrophage polarization to M2 subtype. Frontiers in Immunology 9 3142. (https://doi.org/10.3389/fimmu.2018.03142)

Meggyes M, Miko E, Polgar B, Bogar B, Farkas B, Illes Z \& Szereday L 2014 Peripheral blood TIM-3 positive NK and CD8+ T cells throughout pregnancy: TIM-3/galectin-9 interaction and its possible role during pregnancy. PLOS ONE 9 e92371. (https://doi.org/10.1371/journal. pone.0092371)

Moffett A \& Loke C 2006 Immunology of placentation in eutherian mammals. Nature Reviews: Immunology 6 584-594. (https://doi. org/10.1038/nri1897)

O'Brien MJ, Shu Q, Stinson WA, Tsou PS, Ruth JH, Isozaki T, Campbell PL, Ohara RA, Koch AE, Fox DA et al. 2018 A unique role for galectin-9 in angiogenesis and inflammatory arthritis. Arthritis Research and Therapy 20 31. (https://doi.org/10.1186/s13075-018-1519-x)

Popovici RM, Krause MS, Germeyer A, Strowitzki T \& von Wolff M 2005 Galectin-9: a new endometrial epithelial marker for the midand late-secretory and decidual phases in humans. Journal of Clinical Endocrinology and Metabolism 90 6170-6176. (https://doi.org/10.1210/ jc.2004-2529)

Shennan AH, Green M \& Chappell LC 2017 Maternal deaths in the UK: pre-eclampsia deaths are avoidable. Lancet 389 582-584. (https://doi. org/10.1016/S0140-6736(17)30184-8)
Sheu A, Chan Y, Ferguson A, Bakhtyari MB, Hawke W, White C, Chan YF, Bertolino PJ, Woon HG, Palendira U et al. 2018 A proinflammatory CD4(+) T cell phenotype in gestational diabetes mellitus. Diabetologia 61 1633-1643.

Sun J, Yang M, Ban Y, Gao W, Song B, Wang Y, Zhang Y, Shao Q, Kong B \& Qu X 2016 Tim-3 is upregulated in NK cells during early pregnancy and inhibits NK cytotoxicity toward trophoblast in galectin-9 dependent pathway. PLOS ONE 11 e0147186. (https://doi.org/10.1371/journal. pone.0147186)

Svensson-Arvelund J, Mehta RB, Lindau R, Mirrasekhian E, RodriguezMartinez H, Berg G, Lash GE, Jenmalm MC \& Ernerudh J 2015 The human fetal placenta promotes tolerance against the semiallogeneic fetus by inducing regulatory T cells and homeostatic M2 macrophages. Journal of Immunology 194 1534-1544. (https://doi.org/10.4049/ jimmunol.1401536)

Wang S, Cao C, Piao H, Li Y, Tao Y, Zhang X, Zhang D, Sun C, Zhu R \& Wang Y 2015 Tim-3 protects decidual stromal cells from toll-like receptor-mediated apoptosis and inflammatory reactions and promotes Th2 bias at the maternal-fetal interface. Scientific Reports 59013. (https://doi.org/10.1038/srep09013)

Wang S, Sun F, Li M, Qian J, Chen C, Wang M, Zang X, Li D, Yu M \& Du M 2019 The appropriate frequency and function of decidual Tim-3(+)CTLA$4(+)$ CD8(+) T cells are important in maintaining normal pregnancy. Cell Death and Disease 10 407. (https://doi.org/10.1038/s41419-0191642-x)

Received 11 November 2019

First decision 18 December 2019

Revised Manuscript received 24 September 2020

Accepted 2 October 2020 